$11-2014$

\title{
Estimation of Multi Component Systems Reliability in Stress-Strength Models
}

\author{
Adil H. Khan \\ University of Kashmir, Srinagar, India, khanadil_192@yahoo.com \\ T R.Jan \\ University of Kashmir, Srinagar, India, drtrjan@gmail.com
}

Follow this and additional works at: http://digitalcommons.wayne.edu/jmasm

Part of the Applied Statistics Commons, Social and Behavioral Sciences Commons, and the Statistical Theory Commons

\section{Recommended Citation}

Khan, Adil H. and Jan, T R. (2014) "Estimation of Multi Component Systems Reliability in Stress-Strength Models," Journal of Modern Applied Statistical Methods: Vol. 13 : Iss. 2 , Article 21.

DOI: $10.22237 /$ jmasm/1414815600

Available at: http://digitalcommons.wayne.edu/jmasm/vol13/iss2/21

This Regular Article is brought to you for free and open access by the Open Access Journals at DigitalCommons@WayneState. It has been accepted for inclusion in Journal of Modern Applied Statistical Methods by an authorized editor of DigitalCommons@WayneState. 


\section{Estimation of Multi Component Systems Reliability in Stress-Strength Models}

\author{
Adil Hamid Khan \\ University of Kashmir \\ Srinagar, India
}

\author{
T.R. Jan \\ University of Kashmir \\ Srinagar, India
}

In a system with standby redundancy, there are a number of components only one of which works at a time and the other remain as standbys. When an impact of stress exceeds the strength of the active component, for the first time, it fails and another from standbys, if there is any, is activated and faces the impact of stresses, not necessarily identical as faced by the preceding component and the system fails when all the components have failed. Sriwastav and Kakaty (1981) assumed that the components stress-strengths are similarly distributed. However, in general the stress distributions will be different from the strength distributions not only in parameter values but also in forms, because stresses are independent of strengths and the two are governed by different physical conditions. Assume the components in the system for both stress and strength are independent and follow different probability distributions viz. Exponential, Gamma, Lindley. Different conditions for stress and strength were considered. Under these assumptions the reliabilities of the system have been obtained with the help of the particular forms of density functions of $n$-standby system when all stress-strengths are random variables. The expressions for the marginal reliabilities $\mathrm{R}(1), \mathrm{R}(2), \mathrm{R}(3)$ etc. have been obtained based on its stress-strength models. Results obtained by J. Gogoi and M. Bohra are particular case presentations.

Keywords: $\quad$ Reliability, standby redundancy, exponential distribution, Lindley distribution.

\section{Introduction}

The reliability of a system is the probability that when operating under stated environmental conditions, the system will perform its intended function adequately. For stress-strength models both the strength of the system, $X$, and the stress, $Y$, imposed on it by its operating environments are considered to be random variables.

Adil H. Khan is a Research Scholar in the Post-Graduate Department of Statistics. Email him khanadil_192@yahoo.com. T. R. Jan is an Assistant Professor in the Post-Graduate Department of Statistics. Email him at drtrjan@gmail.com. 


\section{ESTIMATION OF MULTI COMPONENT SYSTEMS RELIABILITY}

The reliability, $R$, of the system is the probability that the system is strong enough to overcome the stress imposed on it, that is to say $R=\operatorname{Pr}(X>Y)$.

In a standby system, that is, a system with standby redundancy, there are number of components only one of which works at a time and the other remains as standby. When an impact of stress exceeds the strength of the active component, for the first time, it fails and the another component from standbys, if there is any, is activated and faces the impact of stresses, not necessarily identical as faced by the preceding component. The system fails when all the components have failed. This problem has a long history starting with the pioneering work of Birnbaum (1956) and Birnbaum and McCarty (1958). The term stress-strength was first introduced by Church and Harris (1970). Since then significant amount of work has been done both from parametric and non-parametric point of view. A comprehensive treatment of the different stress-strength models till 2001 can be found in the excellent monograph by Kotz et al. (2003). Some of the recent work on the stress-strength model can be obtained in Kundu and Gupta $(2005,2006)$, Raqab and Kundu (2005), Krishnamoorthy et al. (2007). Gogoi, Borah and Sriwastav (2010) gave an interference model with number of stress a Poisson Process. Gogoi and Borah (2012) obtained the reliability expressions when the stress-strength of the components follow different distributions.

It has been assumed the components stress-strengths are similarly distributed but in general the stress distributions will be different from the strength distributions not only in parameter values but also in forms because stresses are independent of strengths and the two are governed by different physical conditions. It is assumed here that stress-strengths of all the components in the system are independent and obtained the stress-strength parameter $R=\operatorname{Pr}(X>Y)$, when $X$ and $Y$ are independent. Note that the stress-strength parameter plays an important role in the reliability analysis. For example if $X$ is the strength of a system which is subjected to stress $Y$, then the parameter $R$ measures the system performance and it is very common in the context of mechanical reliability of a system. Moreover, $R$ provides the probability of a system failure, if the system fails whenever the applied stress is greater than its strength.

\section{Model}

Consider an n-standby system in which, initially, there are $\mathrm{n}$ components, out of which only one is working under impact of stresses and the remaining $(n-1)$ are standby. Whenever the working component fails, one from the standby takes its place and is subjected to impact of stresses and the system works. The system fails when the entire component fails. 


\section{KHAN \& JAN}

Let $X_{1}, X_{2}, \ldots, X_{n}$ be a set of $\mathrm{n}$ independent random variables, representing the strengths of n components arranged in order of activation in the system and let $Y_{1}, Y_{2}, \ldots, Y_{n}$, be another set of independent random variables representing the stresses on the n components respectively, then the system reliability $R_{n}$ of the system is given by

$$
R_{n}=R(1)+R(2)+\ldots . . R(n)
$$

where the marginal reliability $R(r)$ is the contribution to the reliability of the system by the $\mathrm{r}^{\text {th }}$ component and is defined as

$$
R(r)=\operatorname{Pr}\left[X_{1}<Y_{1} X_{2}<Y_{2}, \ldots, X_{r-1}<Y_{r-1} \geq Y_{r}\right]
$$

and if $f_{i}(x)$ and $h_{i}(y)$ are the probability density functions of $X_{i}$ and $Y_{i}, i=1,2,3, \ldots, n$ respectively then

$$
\begin{aligned}
R(r)= & {\left[\int_{-\infty}^{\infty} F_{1}(y) h_{1}(y) d y\right]\left[\int_{-\infty}^{\infty} F_{2}(y) h_{2}(y) d y\right] } \\
& \ldots\left[\int_{-\infty}^{\infty} F_{r-1}(y) h_{r-1}(y) d y\right]\left[\int_{-\infty}^{\infty} \bar{F}_{r}(y) h_{r}(y) d y\right]
\end{aligned}
$$

where $F_{i}(y)$ is the commutative distribution function of $X_{i}$ and

$$
\bar{F}_{i}(y)=1-F(x)
$$

Assume that strength and stress follow different distributions, the following cases are considered.

(I) One parameter exponential strength and three parameter exponential stress.

(II) Two parameter Lindley strength and one parameter gamma stress.

(III) Two parameter Lindley strength and two parameter gamma stress. 


\section{ESTIMATION OF MULTI COMPONENT SYSTEMS RELIABILITY}

\section{One parameter exponential strength and three parameter exponential stress}

Let $f_{i}(x)$ be the one parameter exponential strength with parameter $\lambda_{i}$ and $h_{i}(x)$ be the three parameter exponential stress with parameters $\alpha_{i}, \beta_{i}$ and $\mu_{i} ; i=1,2, \ldots, n$, then

$$
f_{i}(x, \lambda)=\left\{\begin{array}{c}
\lambda_{i} e^{-\lambda_{i} x_{i}} ; x_{i} \geq 0, \lambda_{i} \geq 0 \\
0 ; \text { otherwise }
\end{array}\right.
$$

and

$$
h_{i}(y, \alpha, \beta, \mu)=\left\{\begin{array}{c}
\frac{\alpha_{i}}{\beta_{i}}\left(1-e^{-\left(y_{i}-\mu_{i}\right) / \beta_{i}}\right) ; \quad y_{i}>\mu_{i} \alpha_{i}>0, \beta_{i}>0 \\
0 ; \text { otherwise }
\end{array}\right.
$$

Then from equation (2)

$$
\begin{aligned}
R(1)= & \int_{\mu_{1}}^{\infty} \bar{F}_{1}(y) h_{1}(y) d y=\frac{\alpha_{1} e^{-\lambda_{1} \mu_{1}} \Gamma\left(\alpha_{1}\right) \Gamma\left(\lambda_{1} \beta_{1}+1\right)}{\Gamma\left(\alpha_{1}+\lambda_{1} \beta_{1}+1\right)} \\
R(2)= & {\left[\int_{\mu_{1}}^{\infty} F_{1}(y) h_{1}(y) d y\right]\left[\int_{\mu_{2}}^{\infty} \bar{F}_{2}(y) h_{2}(y) d y\right] } \\
& =\left[1-\frac{\alpha_{1} e^{-\lambda_{1} \mu_{1}} \Gamma\left(\alpha_{1}\right) \Gamma\left(\lambda_{1} \beta_{1}+1\right)}{\Gamma\left(\alpha_{1}+\lambda_{1} \beta_{1}+1\right)}\right]\left[\frac{\alpha_{2} e^{-\lambda_{2} \mu_{2}} \Gamma\left(\alpha_{2}\right) \Gamma\left(\lambda_{2} \beta_{2}+1\right)}{\Gamma\left(\alpha_{2}+\lambda_{2} \beta_{2}+1\right)}\right] \\
R(3)= & {\left[\int_{\mu_{1}}^{\infty} F_{1}(y) h_{1}(y) d y\right]\left[\int_{\mu_{2}}^{\infty} F_{2}(y) h_{2}(y) d y\right]\left[\int_{\mu_{3}}^{\infty} \bar{F}_{3}(y) h_{3}(y) d y\right] } \\
= & {\left[1-\frac{\alpha_{1} e^{-\lambda_{1} \mu_{1}} \Gamma\left(\alpha_{1}\right) \Gamma\left(\lambda_{1} \beta_{1}+1\right)}{\Gamma\left(\alpha_{1}+\lambda_{1} \beta_{1}+1\right)}\right]\left[1-\frac{\alpha_{2} e^{-\lambda_{2} \mu_{2}} \Gamma\left(\alpha_{2}\right) \Gamma\left(\lambda_{2} \beta_{2}+1\right)}{\Gamma\left(\alpha_{2}+\lambda_{2} \beta_{2}+1\right)}\right] } \\
& \times\left[\frac{\alpha_{3} e^{-\lambda_{3} \mu_{3}} \Gamma\left(\alpha_{3}\right) \Gamma\left(\lambda_{3} \beta_{3}+1\right)}{\Gamma\left(\alpha_{3}+\lambda_{3} \beta_{3}+1\right)}\right]
\end{aligned}
$$


In general,

$$
\begin{aligned}
R(r) & =\left[1-\frac{\alpha_{1} e^{-\lambda_{1} \mu_{1}} \Gamma\left(\alpha_{1}\right) \Gamma\left(\lambda_{1} \beta_{1}+1\right)}{\Gamma\left(\alpha_{1}+\lambda_{1} \beta_{1}+1\right)}\right]\left[1-\frac{\alpha_{2} e^{-\lambda_{2} \mu_{2}} \Gamma\left(\alpha_{2}\right) \Gamma\left(\lambda_{2} \beta_{2}+1\right)}{\Gamma\left(\alpha_{2}+\lambda_{2} \beta_{2}+1\right)}\right] \ldots \\
& \times\left[1-\frac{\alpha_{r-1} e^{-\lambda_{r-1} \mu_{r-1}} \Gamma\left(\alpha_{r-1}\right) \Gamma\left(\lambda_{r-1} \beta_{r-1}+1\right)}{\Gamma\left(\alpha_{r-1}+\lambda_{r-1} \beta_{r-1}+1\right)}\right]\left[\frac{\alpha_{r} e^{-\lambda_{r} \mu_{r}} \Gamma\left(\alpha_{r}\right) \Gamma\left(\lambda_{r} \beta_{r}+1\right)}{\Gamma\left(\alpha_{r}+\lambda_{r} \beta_{r}+1\right)}\right] .(6)
\end{aligned}
$$

\section{Particular Case}

When in (4) $\alpha_{\mathrm{i}}=1$, the three parameter exponential distribution becomes two parameter exponential distribution. Then the expression ${ }^{R(r)}$ for one parameter exponential strength and two parameter exponential stress is obtained from (6) by putting $\alpha_{i}=1 ; i=1,2, \ldots, r$ and is given by

$$
R(r)=\left[1-\frac{e^{-\lambda_{1} \mu_{1}}}{\lambda_{1} \beta_{1}+1}\right]\left[1-\frac{e^{-\lambda_{2} \mu_{2}}}{\lambda_{2} \beta_{2}+1}\right] \ldots\left[1-\frac{e^{-\lambda_{r-1} \mu_{r-1}}}{\lambda_{r-1} \beta_{r-1}+1}\right]\left[\frac{e^{-\lambda_{r} \mu_{r}}}{\lambda_{r} \beta_{r}+1}\right]
$$

The result in (7) is obtained by Gogoi and Borah (2012)

\section{Two parameter Lindley strength and one parameter gamma stress}

Let $f_{i}(x)$ be the two parameter Lindley strength with parameters $\theta_{\mathrm{i}}$ and $\alpha_{\mathrm{i}}$ and $h_{i}(x)$ be the one parameter gamma stress with parameter $m_{i}, i=1,2, \ldots, n$, the

$$
f_{i}(x, \theta, \alpha)=\left\{\begin{array}{c}
\frac{\theta_{i}^{2}}{\theta_{i}+\alpha_{i}}\left(1+\theta_{i} x_{i}\right) e^{-\theta_{i} x_{i}} ; x_{i} \geq 0, \theta_{i}>0, \alpha_{i}>-\theta_{i} \\
0 ; \text { otherwise }
\end{array}\right.
$$

and

$$
h_{i}(y, m)=\left\{\begin{array}{c}
\frac{1}{\Gamma\left(m_{i}\right)} e^{-y_{i}} y_{i}^{m_{i}} ; y_{i} \geq 0, m_{i} \geq 1 \\
0 ; \text { otherwise }
\end{array}\right.
$$




\section{ESTIMATION OF MULTI COMPONENT SYSTEMS RELIABILITY}

Then from equation (2)

$$
\begin{aligned}
& R(1)=\int_{0}^{\infty} \bar{F}_{1}(y) h_{1}(y) d y=\frac{\left(\theta_{1}+\alpha_{1}\right)\left(\theta_{1}+1\right)+\alpha_{1} \theta_{1} m_{1}}{\left(\theta_{1}+\alpha_{1}\right)\left(\theta_{1}+1\right)^{m_{1}+1}} \\
& R(2)=\left[\int_{0}^{\infty} F_{1}(y) h_{1}(y) d y\right]\left[\int_{0}^{\infty} \bar{F}_{2}(y) h_{2}(y) d y\right] \\
& =\left[1-\frac{\left(\theta_{1}+\alpha_{1}\right)\left(\theta_{1}+1\right)+\alpha_{1} \theta_{1} m_{1}}{\left(\theta_{1}+\alpha_{1}\right)\left(\theta_{1}+1\right)^{m_{1}+1}}\right]\left[\frac{\left(\theta_{2}+\alpha_{2}\right)\left(\theta_{2}+1\right)+\alpha_{2} \theta_{2} m_{2}}{\left(\theta_{2}+\alpha_{2}\right)\left(\theta_{2}+1\right)^{m_{2}+1}}\right] \\
& R(3)=\left[\int_{0}^{\infty} F_{1}(y) h_{1}(y) d y\right]\left[\int_{0}^{\infty} F_{2}(y) h_{2}(y) d y\right]\left[\int_{0}^{\infty} \bar{F}_{3}(y) h_{3}(y) d y\right] \\
& =\left[1-\frac{\left(\theta_{1}+\alpha_{1}\right)\left(\theta_{1}+1\right)+\alpha_{1} \theta_{1} m_{1}}{\left(\theta_{1}+\alpha_{1}\right)\left(\theta_{1}+1\right)^{m_{1}+1}}\right]\left[1-\frac{\left(\theta_{2}+\alpha_{2}\right)\left(\theta_{2}+1\right)+\alpha_{2} \theta_{2} m_{2}}{\left(\theta_{2}+\alpha_{2}\right)\left(\theta_{2}+1\right)^{m_{2}+1}}\right] \\
& \times\left[\frac{\left(\theta_{3}+\alpha_{3}\right)\left(\theta_{3}+1\right)+\alpha_{3} \theta_{3} m_{3}}{\left(\theta_{3}+\alpha_{3}\right)\left(\theta_{3}+1\right)^{m_{3}+1}}\right]
\end{aligned}
$$

In general

$$
\begin{aligned}
R(r)= & {\left[1-\frac{\left(\theta_{1}+\alpha_{1}\right)\left(\theta_{1}+1\right)+\alpha_{1} \theta_{1} m_{1}}{\left(\theta_{1}+\alpha_{1}\right)\left(\theta_{1}+1\right)^{m_{1}+1}}\right]\left[1-\frac{\left(\theta_{2}+\alpha_{2}\right)\left(\theta_{2}+1\right)+\alpha_{2} \theta_{2} m_{2}}{\left(\theta_{2}+\alpha_{2}\right)\left(\theta_{2}+1\right)^{m_{2}+1}}\right] \ldots } \\
& {\left[1-\frac{\left(\theta_{r-1}+\alpha_{r-1}\right)\left(\theta_{r-1}+1\right)+\alpha_{r-1} \theta_{r-1} m_{r-1}}{\left(\theta_{r-1}+\alpha_{r-1}\right)\left(\theta_{r-1}+1\right)^{m_{r-1}+1}}\right]\left[\frac{\left(\theta_{r}+\alpha_{r}\right)\left(\theta_{r}+1\right)+\alpha_{r} \theta_{r} m_{r}}{\left(\theta_{r}+\alpha_{r}\right)\left(\theta_{r}+1\right)^{m_{r}+1}}\right] }
\end{aligned}
$$

\section{Particular case}

1) When $\alpha_{\mathrm{i}}=1$ in (8) two parameter Lindley distribution becomes one parameter Lindley distribution. Then the expression $R(r)$ for one parameter Lindley strength and one parameter gamma stress is obtained from (10) by putting $\alpha_{i}=1 ; i=1,2, \ldots, r$ and is given by 


$$
\begin{aligned}
R(r)= & {\left[1-\frac{\left(\theta_{1}+1\right)\left(\theta_{1}+1\right)+\theta_{1} m_{1}}{\left(\theta_{1}+1\right)\left(\theta_{1}+1\right)^{m_{1}+1}}\right]\left[1-\frac{\left(\theta_{2}+1\right)\left(\theta_{2}+1\right)+\theta_{2} m_{2}}{\left(\theta_{2}+1\right)\left(\theta_{2}+1\right)^{m_{2}+1}}\right] \ldots } \\
& {\left[1-\frac{\left(\theta_{r-1}+1\right)\left(\theta_{r-1}+1\right)+\theta_{r-1} m_{r-1}}{\left(\theta_{r-1}+1\right)\left(\theta_{r-1}+1\right)^{m_{r-1}+1}}\right]\left[\frac{\left(\theta_{r}+1\right)\left(\theta_{r}+1\right)+\theta_{r} m_{r}}{\left(\theta_{r}+1\right)\left(\theta_{r}+1\right)^{m_{r}+1}}\right] }
\end{aligned}
$$

(The result in (11) is obtained by Gogoi and Borah (2012))

2) When $\alpha_{i}=0$ in (8) two parameter Lindley distribution becomes one parameter exponential distribution. Then the expression $R(r)$ for one parameter exponential strength and one parameter gamma stress is obtained from (10) by putting $\alpha_{i}=1 ; i=1,2, \ldots, r$ and is given by

$$
R(r)=\left[1-\frac{1}{\left(\theta_{1}+1\right)^{m_{1}}}\right]\left[1-\frac{1}{\left(\theta_{2}+1\right)^{m_{2}}}\right] \ldots\left[1-\frac{1}{\left(\theta_{r-1}+1\right)^{m_{r-1}}}\right]\left[\frac{1}{\left(\theta_{r}+1\right)^{m_{r}}}\right]
$$

\section{Two parameter Lindley strength and two parameter gamma stress}

Let $f_{i}(x)$ be the two parameter Lindley strength with parameters $\theta_{i}$ and $\alpha_{i}$ and $h_{i}(x)$ be the two parameter gamma stress with parameter $m_{i}$ and $\mu_{i}, i=1,2, \ldots, n$, then

$$
f_{i}(x, \theta, \alpha)=\left\{\begin{array}{c}
\frac{\theta_{i}^{2}}{\theta_{i}+\alpha_{i}}\left(1+\theta_{i} x_{i}\right) e^{-\theta_{i} x_{i}} ; x_{i} \geq 0, \theta_{i}>0, \alpha_{i}>-\theta_{i} \\
0 ; \text { otherwise }
\end{array}\right.
$$

and

$$
h_{i}(y, m, \mu)=\left\{\begin{aligned}
\frac{1}{\mu_{i}^{m_{i}} \Gamma\left(m_{i}\right)} e^{\frac{-y_{i}}{\mu_{i}}} y_{i}^{m_{i}-1} ; y_{i}, m_{i}, \mu_{i}>0 \\
0 ; \quad \text { otherwise }
\end{aligned}\right.
$$




\section{ESTIMATION OF MULTI COMPONENT SYSTEMS RELIABILITY}

Then from equation (2)

$$
\begin{aligned}
& R(1)=\int_{0}^{\infty} \bar{F}_{1}(y) h_{1}(y) d y=\frac{\left(\theta_{1}+\alpha_{1}\right)\left(\theta_{1} \mu_{1}+1\right)+\alpha_{1} \theta_{1} m_{1} \mu_{1}}{\left(\theta_{1}+\alpha_{1}\right)\left(\theta_{1} \mu_{1}+1\right)^{m_{1}+1}} \\
& R(2)=\left[\int_{0}^{\infty} F_{1}(y) h_{1}(y) d y\right]\left[\int_{0}^{\infty} \bar{F}_{2}(y) h_{2}(y) d y\right] \\
& =\left[1-\frac{\left(\theta_{1}+\alpha_{1}\right)\left(\theta_{1} \mu_{1}+1\right)+\alpha_{1} \theta_{1} m_{1} \mu_{1}}{\left(\theta_{1}+\alpha_{1}\right)\left(\theta_{1} \mu_{1}+1\right)^{m_{1}+1}}\right]\left[\frac{\left(\theta_{2}+\alpha_{2}\right)\left(\theta_{2} \mu_{2}+1\right)+\alpha_{2} \theta_{2} m_{2} \mu_{2}}{\left(\theta_{2}+\alpha_{2}\right)\left(\theta_{2} \mu_{2}+1\right)^{m_{2}+1}}\right] \\
& R(3)=\left[\int_{0}^{\infty} F_{1}(y) h_{1}(y) d y\right]\left[\int_{0}^{\infty} F_{2}(y) h_{2}(y) d y\right]\left[\int_{0}^{\infty} \bar{F}_{3}(y) h_{3}(y) d y\right] \\
& =\left[1-\frac{\left(\theta_{1}+\alpha_{1}\right)\left(\theta_{1} \mu_{1}+1\right)+\alpha_{1} \theta_{1} m_{1} \mu_{1}}{\left(\theta_{1}+\alpha_{1}\right)\left(\theta_{1} \mu_{1}+1\right)^{m_{1}+1}}\right]\left[1-\frac{\left(\theta_{2}+\alpha_{2}\right)\left(\theta_{2} \mu_{2}+1\right)+\alpha_{2} \theta_{2} m_{2} \mu_{2}}{\left(\theta_{2}+\alpha_{2}\right)\left(\theta_{2} \mu_{2}+1\right)^{m_{2}+1}}\right] \\
& \times\left[\frac{\left(\theta_{3}+\alpha_{3}\right)\left(\theta_{3} \mu_{3}+1\right)+\alpha_{3} \theta_{3} m_{3} \mu_{3}}{\left(\theta_{3}+\alpha_{3}\right)\left(\theta_{3} \mu_{3}+1\right)^{m_{3}+1}}\right]
\end{aligned}
$$

In general,

$$
\begin{aligned}
& R(r)=\left[1-\frac{\left(\theta_{1}+\alpha_{1}\right)\left(\theta_{1} \mu_{1}+1\right)+\alpha_{1} \theta_{1} m_{1} \mu_{1}}{\left(\theta_{1}+\alpha_{1}\right)\left(\theta_{1} \mu_{1}+1\right)^{m_{1}+1}}\right]\left[1-\frac{\left(\theta_{2}+\alpha_{2}\right)\left(\theta_{2} \mu_{2}+1\right)+\alpha_{2} \theta_{2} m_{2} \mu_{2}}{\left(\theta_{2}+\alpha_{2}\right)\left(\theta_{2} \mu_{2}+1\right)^{m_{2}+1}}\right] \ldots \\
& {\left[1-\frac{\left(\theta_{r-1}+\alpha_{r-1}\right)\left(\theta_{r-1} \mu_{r-1}+1\right)+\alpha_{r-1} \theta_{r-1} m_{r-1} \mu_{r-1}}{\left(\theta_{r-1}+\alpha_{r-1}\right)\left(\theta_{r-1} \mu_{r-1}+1\right)^{m_{r-1}+1}}\right]\left[\frac{\left(\theta_{r}+\alpha_{r}\right)\left(\theta_{r} \mu_{r}+1\right)+\alpha_{r} \theta_{r} m_{r} \mu_{r}}{\left(\theta_{r}+\alpha_{r}\right)\left(\theta_{r} \mu_{r}+1\right)^{m_{r}+1}}\right]}
\end{aligned}
$$

\section{Particular Case}

1) When $\alpha_{\mathrm{i}}=1$ in (13) two parameter Lindley distribution becomes one parameter Lindley distribution. Then the expression $R(r)$ for one parameter Lindley strength and two parameter gamma stress is obtained from (15) by putting $\alpha_{i}=1 ; i=1,2, \ldots, r$ and is given by 


$$
\begin{aligned}
R(r)= & {\left[1-\frac{\left(\theta_{1}+1\right)\left(\theta_{1} \mu_{1}+1\right)+\theta_{1} m_{1} \mu_{1}}{\left(\theta_{1}+1\right)\left(\theta_{1} \mu_{1}+1\right)^{m_{1}+1}}\right]\left[1-\frac{\left(\theta_{2}+1\right)\left(\theta_{2} \mu_{2}+1\right)+\theta_{2} m_{2} \mu_{2}}{\left(\theta_{2}+1\right)\left(\theta_{2} \mu_{2}+1\right)^{m_{2}+1}}\right] \ldots } \\
& {\left[1-\frac{\left(\theta_{r-1}+1\right)\left(\theta_{r-1} \mu_{r-1}+1\right)+\theta_{r-1} m_{r-1} \mu_{r-1}}{\left(\theta_{r-1}+1\right)\left(\theta_{r-1} \mu_{r-1}+1\right)^{m_{r-1}+1}}\right]\left[\frac{\left(\theta_{r}+1\right)\left(\theta_{r} \mu_{r}+1\right)+\theta_{r} m_{r} \mu_{r}}{\left(\theta_{r}+1\right)\left(\theta_{r} \mu_{r}+1\right)^{m_{r}+1}}\right] }
\end{aligned}
$$

2) When $\alpha_{i}=0$ in (13) two parameter Lindley distribution becomes one parameter exponential distribution. Then the expression $\mathrm{R}(\mathrm{r})$ for one parameter exponential strength and two parameter gamma stress is obtained from (15) by putting $\alpha_{i}=1 ; i=1,2, \ldots, r$ and is given by

$$
R(r)=\left[1-\frac{1}{\left(\theta_{1} \mu_{1}+1\right)^{m_{1}}}\right]\left[1-\frac{1}{\left(\theta_{2} \mu_{2}+1\right)^{m_{2}}}\right] \ldots\left[1-\frac{1}{\left(\theta_{r-1} \mu_{r-1}+1\right)^{m_{r-1}}}\right]\left[\frac{1}{\left(\theta_{r} \mu_{r}+1\right)^{m_{r}}}\right]
$$

The results in (16) and (17) were obtained by Gogoi and Borah (2012)

\section{References}

Birnbaum, Z. W. (1956). On a use of the Mann-Whitney Statistics, Proceedings of the 3rd Berkley symposium I, 13-17.

Birnbaum, Z. W., \& McCarty, R. C. (1958). A distribution-free upper confidence bound for $\operatorname{Pr}\{\mathrm{Y}<\mathrm{X}\}$, based on independent samples of $\mathrm{X}$ and $\mathrm{Y}$. The Annals of Mathematical Statistics, 29(2), 558-562.

Church, J. D., \& Harris, B. (1970). The estimation of reliability from stressstrength relationships. Technometrics, 12, 49-54.

Ghitany, M. E., Atieh, B., \& Nadarajah, S. (2008). Lindley distribution and its applications. Mathematical and Computers in Simulations, 78, 493-504.

Gogoi, J., Borah, M., \& Sriwastav, G. L. (2010). An interference model with number of stresses a Poisson process. IAPQR Transactions, 34(2), 139-152.

Gogoi, J., \& Borah, M. (2012). Estimation of reliability for multicomponent systems using exponential Gamma and Lindley stress-strength distributions. Journal of Reliability and Statistics Studies, 5(1), 33-41.

Kakari, M. C. (1983). Interference theory and reliability. (Unpublished doctoral dissertation). Dibrugarh University, India. 


\section{ESTIMATION OF MULTI COMPONENT SYSTEMS RELIABILITY}

Kotz, S., Lumelskii, Y., \& Pensky, M. (2003). The Stress-Strength Model and its Generalizations: Theory and Applications. Singapore: World Scientific Press.

Krishnamoorthy, K., Shubhabarata, M., \& Guo, H. (2007). Inference on Reliability in two parameter exponential stress-strength model, Metrika, 65(3), 261-273.

Kundu, D., \& Gupta, R. D. (2005). Estimation of $\mathrm{P}[\mathrm{Y}<\mathrm{X}]$ for generalized exponential distribution. Metrika, 61, 291-308.

Kundu, D., \& Gupta, R. D. (2006). Estimation of R = P[Y < X] for Weibull distributions. IEEE Transactions on Reliability, 55, 270-280.

Pandit, S. N. N., \& Sriwastav, G. L. (1975). Studies in Cascade Reliability-I, IEEE Transactions on Reliability, 24(1), 53-56.

Raqab, M. Z., \& Kundu, D. (2005). Comparison of Different Estimators of $\mathrm{P}[\mathrm{Y}<\mathrm{X}]$ for a Scaled Burr Type X Distribution. Communications in StatisticsSimulation and Computation, 34, 465-483.

Sriwastav, G. L., \& Kakati, M. C. (1981). A stress-strength model with redundancy. IAPQR Transactions, 6(1), 21-27. 